\title{
Implication of Serum and Salivary Levels of Albumin as a Diagnostic Tool in Oral Premalignancy and Malignancy
}

\author{
Riti Tushar Kanti Sinha
}

Department of Pathology, ESI-PGIMSR Medical College \& Hospital, Joka, Kolkata, West Bengal, India

\begin{abstract}
Background: Reactive oxygen and nitrogen species are involved in the initiation and promotion of the multistep carcinogenesis. In neoplastic conditions many biochemical substances many biochemical substances including albumin show altered values .The aim of the present study was to investigate whether serum and salivary albumin levels can be used as diagnostic tools in oral premalignant and malignant leisons.

Methods: The study includes a total of 45 subjects with 15 each of normal healthy, pre-malignancy and malignancy. Fivemilliliter of venous blood was drawn and unstimulated saliva was collected in the early morning. Albumin levels were estimated by Bromocresol Green Method.

Results: Serum albumin levels were found to decrease in pre-malignancy and malignancy as compared to healthy individuals. Salivary Albumin levels were increased in pre-malignancy and malignancy as compared to healthy individuals.

Conclusion: Tobacco consumption is one of the most important risk factors for the development of oral cancer. Burst of reactive oxygen species which cause oxidative stress in tobacco users is prime form of damage brought to the genetic material leading to non-lethal mutations causing pre-malignancy and malignancy.Serum protein has long been implicated to have anti-oxidant properties due to rich concentration of free thiol group. Albumin is a potent extracellular anti oxidant defense and a reliable marker of oxidative stress in the body. It could be helpful in the early identification of various oral pre malignancy and malignant leisons.
\end{abstract}

Keywords: Albumin, Serum, Salivary, Oral Malignancy

\section{Introduction}

Oral cancer is a major public health problem in Indian subcontinent. It ranks among the top three types of cancer in the country. Oral cancer has much higher prevalence among the elderly people as compared to younger population. This might be result of age related increase in magnitude of the attack of free radicals including the reactive oxygen and nitrogen species causing various DNA mutation and/or, aberrations, and reduction in the body's antioxidant defenses.[1] Reactive oxygen species (ROS) or oxygen free radicals are products of normal cellular metabolism[2,3,4]. Oxidative stress (OS) can be defined as an imbalance between the production of highly reactive molecular species and antioxidant defense systems. The most common free oxygen radicals include hydroxyl (HO), nitric oxide (NO), superoxide (O2 -), hydrogen peroxide (H2O2) and peroxynitrite (ONOO-) $[5,6]$.

Plasma levels of anti-oxidants are reliable indicators of the antioxidant status, because they reflect the bioavailability as well as increased utilization of anti-oxidants to counter oxidative stress. The level is also influenced by tobacco and alcohol. Serum proteins have long been implicated to have antioxidant properties owing to their free group.
Among them Albumin is seen as the most potent and abundant extra-cellular antioxidant. On the other hand saliva has long been viewed as a unique yet complex body fluid like plasma or serum [7, 8]. Saliva represents first line of defense against oxidative stress and contains various antioxidants like Albumin [9]. The use of saliva as a diagnostic tool presents many advantages: it is easy to collect, non invasive technique can be performed at home, and no special equipment is required for collection, fewer complications [10].

The role of Albumin in the body is convincing enough to be used as a reliable marker of oxidative stress in the body, which could be helpful in early identification and in determining the pre-disposition to various oral precancerous lesions their transformation into frank malignancy $[11,12,13]$.

The role of oxygen free radicals in the initiation, promotion and progression of carcinogenesis and the protective role of anti-oxidants has been subject of much speculation. On literature review, very few studies to the best of our knowledge were found evaluating serum albumin and salivary albumin, in premalignancy and malignancy $[2,10,14,15]$. Hence the present study was planned with 
aim and objective to evaluate and compare serum and salivary albumin levels in normal individuals, patients with pre-malignancy and malignancy.

\section{Materials and Methods}

Source of data: Study was conducted at the OPD of the dental college and IPD of oncology department of ESIC medical college, Joka, Kolkata for a period of eight months, which comprised of three groups. Group one consisted of 15 healthy individuals, Group two consisted of 15 patients diagnosed with leukoplakia, Group three consisted of 15 patients with clinically and histological diagnosed oral squamous cell carcinoma.

Method of collection of data None of the patients' were on any therapeutic modality, or suffering from any systemic conditions, especially hepatic, or renal disorders that could have affected albumin levels in the body. Also, controls as well as the patients who were chronic alcoholics were excluded from the study to rule out the probability of derangement of liver function that could have contributed towards the variations in sera levels of albumin.

Selected patients were explained in detail about the planned study and written informed consents were obtained and ethical clearance certificate was obtained before the study was conducted. Patients were instructed to fast over night. Next day between 8 A.M. and 10 A.M. $5 \mathrm{ml}$ of venous blood was drawn from the selected patients using sterile disposable syringe taking full precautions to prevent haemolysis. Serum was separated by ultracentrifugation taking full precautions to prevent hemolysis. Assay of albumin was carried out with the help of Bromocresol Green Method.

Saliva sample collection: Saliva samples were collected in the morning between 6 am and $12 \mathrm{pm}$. Participants were asked not to eat, drink, or perform any kind of oral hygiene procedures before saliva collection. Just before saliva collection, a cup of water was given to the participant for rinsing. Five minutes after rinsing, participants were asked to spit into a $50-\mathrm{mL}$ sterile plastic tube kept in ice. A maximum of $15-20 \mathrm{ml}$ saliva was collected within 30 minutes.

Determination of salivary albumin levels

After thawing, Albumin level in the samples was measured by Albumin Agappe kit done by bcg method, and the values were obtained.

Statistical Analysis: The data was analyzed by One-way Analysis of Variance (ANOVA) using SPSS Statistical Package for social science version 15. The $\mathrm{P}$ values less than 0.05 were taken to be statistically significant. The normality of data was checked before the statistical analysis was done.

\section{Result}

Serum levels of albumin (normal levels $3.4-5.4 \mathrm{gm} /$ dl )were statistically significant $(p<0.005)$ with mean decreasing from $4.63 \mathrm{gm} / \mathrm{dl}$ in normal individuals(group 1)to $2.99 \mathrm{gm} / \mathrm{dl}$ in patients diagnosed with leukoplakia (group 2) and $2.24 \mathrm{gm} / \mathrm{dl}$ in patients diagnosed with oral squamous cell carcinoma (group 3) as shown in Table1. Salivary levels too were statistically significant with mean values increasing from $0.66 \mathrm{gm} / \mathrm{dl}$ in normal individuals to $0.80 \mathrm{gm} / \mathrm{dl}$ in patients diagnosed with leukoplakia and $0.93 \mathrm{gm} / \mathrm{dl}$ in patients diagnosed with oral squamous cell carcinoma as shown in table2.

Serum and salivary Albumin levels were statistically significant on inter comparison of mean values of normal individual with premalignant and malignant group and also when the mean value of premalignant group was compared with malignant group.

Table 1: Evaluation of serum and salivary albumin levels between Normal Control group and premalignant group.

\begin{tabular}{|c|c|c|c|}
\hline & Group & Number & Mean \pm Std. Deviation \\
\hline \multirow{2}{*}{ Serum Albumin Level } & 1(Normal) & 15 & $4.63 \pm 0.60$ \\
\hline & 2(Pre malignant) & 15 & $2.99 \pm 0.32$ \\
\hline \multirow{2}{*}{ Salivary Albumin Level } & 1 (Normal) & 15 & $0.66 \pm 0.08$ \\
\hline & 2(Pre malignant) & 15 & $0.80 \pm 0.06$ \\
\hline
\end{tabular}

Table 2: Evaluation of serum and salivary albumin level between Normal Control group and Malignant group.

\begin{tabular}{|l|c|c|c|c|}
\hline & Group & Number & Mean \pm Std. Deviation & P value \\
\hline \multirow{2}{*}{ Serum Albumin Level } & 1 (Normal) & 15 & $4.63 \pm 0.60$ & \\
\cline { 2 - 5 } & $3($ OSCC) & 15 & $2.24 \pm 0.52$ & $0.02 *$ \\
\hline \multirow{2}{*}{$\begin{array}{l}\text { Salivary Albumin } \\
\text { Level }\end{array}$} & 1 (Normal) & 15 & $0.66 \pm 0.08$ & \multirow{2}{*}{0.03 * } \\
\cline { 2 - 5 } & 3 (OSCC) & 15 & $0.93 \pm 0.20$ & \\
\hline
\end{tabular}




\section{Discussion}

In aerobic life cycle, Reactive Oxygen Species (ROS) are products of normal cellular metabolism produced in mitochondria ${ }^{[16]}$. The respiratory chain in mitochondria is an important source of ROS, primarily $\mathrm{O}^{-}$and $\mathrm{H} 2 \mathrm{O} 2$. ROS can be generated by external environment, the main sources are: heat, UV light, $\mathrm{X}$ and gamma radiations, therapeutic drugs, chemical substance and pathogenic micro-organism ${ }^{[17]}$. Others sources of ROS are the inflammatory reactions, especially chronic inflammation. Inflammatory cells like activated macrophages and neutrophils release various $\operatorname{ROS}^{[18,19]}$. They are short lived and possesses free electron. It is this free electron which is responsible for the high reactivity of free radicals, which can bind other free radicals or subtract an electron from the surrounding molecules ${ }^{[20,21]}$

To counteract these oxygen free radicals, normal living cells have multiple antioxidant defence mechanism acting in the cascade manner. Antioxidants according to definition given in 1989 by Halliwell and Guttteridge are 'substances which slow down the rate at which something decay because of oxidation'. This definition includes non - enzymatic and enzymatic compounds ${ }^{[22]}$. Various enzymatic antioxidants are Superoxide Dismutase (SOD), Glutathione Peroxidase, Catalase, Glutathione Reductase, Glutathione transferase, whereas non - enzymatic antioxidants include Alpha tocopherol, Ascorbate, Retinoids, Beta - Carotene, Glutathione, Selenium, Albumin, and Uric Acid. The antioxidants neutralize free radicals by donating one of their electrons, ending the electron stealing reaction. The antioxidant nutrients, however does not become a free radical by donating an electron because they are stable in either form Oxidative stress arises either from the overproduction of Reactive Oxygen Species or from the deficiency of anti oxidant defence or repair mechanism of body and results in reversible or irreversible tissue injury ${ }^{23}$. Examples of short term oxidative stresses are ischemia reperfusion injury and acute inflammation.

Increased generation of Reactive Oxygen Species may cause toxic effects by oxidative damage of proteins, lipids, and DNA. Lipids particularly low density lipoproteins are particularly targeted by the attack of free radicals and subjected to lipid peroxidation ${ }^{[24]}$. Free radical mediated lipid per-oxidation has been proposed to be a basic mechanism for occurrence of wide variety of diseases and conditions including oral cancer (Beevi et al. 2006) ${ }^{[25]}$.

It has been proposed that free radicals are involved in both the initiation and promotion stages of carcinogenesis ${ }^{[26]}$. These free radicals have been shown that ROS can cause DNA base alterations, strand breaks; damage to tumor suppressor genes and enhanced expression of protooncogene's and alter anti-oxidant defense mechanism ${ }^{[27]}$.
Various experimental studies prove that various antioxidants neutralize or trap Reactive Oxygen Species act as a cancer preventing agents. This support the role of Reactive Oxygen Species in pathogenesis of cancer. ${ }^{[26,27,28]}$

In human Albumin is the most abundant plasma protein, accounting for $55-60 \%$ of the measured serum protein. It consists of a single polypeptide chain of 585 amino acids with a molecular weight of $66,500 \mathrm{Da}$. The total body albumin pool measures about $3.5-5.0 \mathrm{gm} / \mathrm{kg}$ body weight. The molecule is very flexible and changes shape readily with variations in environmental conditions and with binding of ligands29. It is synthesized in liver and secreted into portal circulation. The usual half -life of Albumin is 20 days. Albumin is proposed to have antioxidant potential. This may be related to the abundance of sulfhydryl (-SH) groups on the Albumin molecule along with free Thiol groups [30,31]. Owing to this it can provide ten times more antioxidant protection as compared to other plasma protein against various Reactive Oxygen Species. Hence, it is involved in scavenging of oxygen free radicals, which are said to be involved in pathogenesis of oral cancer.

In the oral cavity, albumin is regarded as a serum ultra filtrate to the mouth and it may diffuse into the mucosal secretions. Salivary albumin is increased in immunosuppressant, diabetes, and radiotherapy. It may be hypothesized that salivary albumin can be used to assess the integrity of mucosal function in the mouth. Albumin is often used as a marker for the degree of mucositis or inflammation in the oral cavity.

In the present study Serum Albumin Levels statistically decreased from $4.63 \mathrm{~g} / \mathrm{dl}$ in healthy control to $2.99 \mathrm{~g} / \mathrm{dl}$ in oral leukoplakia to $2.24 \mathrm{~g} / \mathrm{dl}$ in oral squamous cell carcinoma as shown in table1, which was in accordance with other studies. ${ }^{[9,10,27]}$ Low serum albumin in patients with oral cancer may partly be due to the effect of cytokines such as interleukin-6 (IL6) and tumor necrosis factor (TNF). These inflammatory mediators are produced by tumor and host cells in malignancies. TNF and IL6 may act both by increasing the local transcapillary escape of albumin in the tumor bed and by decreasing the hepatic synthesis of albumin (Richter et al 2000) ${ }^{[31]}$. Low serum albumin may thus reduce the role of albumin in mopping up free radicals and thus increase the potential for toxic cellular injuries that could trigger the process of carcinogenesis.

Various studies ${ }^{[21,30]}$ have shown diet and nutrition as an etiological factor of oral cancer. Franceschi et al. suggested that inadequate nutrition enhances cancer risk. This was in accordance with a study in India, in which subjects with oral cancer were less likely to consume nutritious diet as compared to control group (Notani et al. 1976) ${ }^{[32]}$. Since 
serum Albumin may be reflection of nutritional status and protein intake (knekt et al. 2000), it can be suggested that low serum Albumin levels are associated with an increased risk of oral cancer (Ko et al. 1986) ${ }^{[33]}$.

Low levels of serum Albumin are indicative of an ongoing systemic response that causes loss of proteins. In studies reviewed by Digant Gupta 2010, it was found out that lower levels of serum Albumin were found to be associated with poor survival rate of cancer patients. It can be used as an independent indicator of the need for aggressive nutrition intervention. Hence, serum Albumin is not only a window into the patient's nutritional status but also a useful factor for predicting patient prognosis. The potential advantage of serum Albumin as pre-treatment prognostic factor in cancer patients is that it is inexpensive, reproducible and powerful: $^{[2,34]}$

Saliva being the first biological fluid met by external substances, it represents the first line of defense against oxidative stress ${ }^{[35]}$. Like plasma, saliva is also rich in antioxidants like Albumin, Uric acid, Ascorbic acid and other antioxidant enzymes. The concentrations of Albumin and Ascorbic acid are lower in saliva than those of serum. This may indicate an active secretion system for salivary antioxidants rather than passive diffuse from the circulation. Stimulated saliva contains a lower concentration of antioxidants but when flow rates are taken into account, the antioxidant capacity is higher than in unstimulated saliva [36]. In the present study, the salivary level of Albumin significantly increased, from $0.66 \mathrm{~g} / \mathrm{dl}$ in healthy control to $0.80 \mathrm{~g} / \mathrm{dl}$ in oral leukoplakia to $0.93 \mathrm{~g} / \mathrm{dl}$ in oral squamous cell carcinoma (Table 2), which was in accordance with the results of other studies. ${ }^{[9,10,15,27]}$. Uric acid and Albumin appear to contribute to most of the antioxidant protection in whole saliva. Uric acid is the principal antioxidant found in saliva. In case of oral precancer and oral cancer level of salivary Uric acid becomes low, and level of salivary Albumin increases ${ }^{[37]}$. So the increase in salivary Albumin level represents a compensatory antioxidant defense system to counteract Albumin has well-established physiological functions in health.

Various other functions of Albumin are as follows ${ }^{[29]}$, in maintaining oncotic pressure, binding of various substances like different drugs, maintenance of metabolic function, acts as a plasma buffer to maintain Acid-Base function and maintaining micro vascular integrity, effect on blood coagulation. Recently nano-particle albumin-bound (nab) paclitaxel (nab-paclitaxel) has attracted much interest in anti-cancer drug delivery system.

The difficulty in measuring oxidative stress with histochemical, spectrophotometric or proteomic and genomic assays and its aetiopathogenic link with pathological conditions are among the reasons that have hold back research and development in this field so far. Therefore recently, new practical and reliable instruments have been set up. They only requirements a drop of blood or a sample of urine or saliva and is able to provide a generic value of the patient's oxidative stress quantifying his amount of free radicals. Furthermore, several other tests, which are not found in the literature, are available in the market. Recently, a new non-invasive instrument (FRAS 4 EVOLVO - Free radical system; H\&D, Parma, Italy), has been introduced in the clinical setting. It analyses a sample of saliva giving the grade of oxidative stress in the oral cavity, and it can be useful for the study of particular pathological conditions affecting this district. Everything has been achieved with the aid of an instrument that allows the measurement of the antioxidant concentration starting from a simple saliva specimen ${ }^{[22]}$.

As this study was carried out in limited population with specific inclusion criteria, sample size of present study is very small, in future large studies with larger sample size is recommended to generalized the results.

\section{Conclusion}

Reactive Oxygen Species have long been implicated in the genesis of oral cancers. Results in the present study suggest the role of albumin in the early diagnosis and impact on the prognosis of premalignant and malignant patients. It provides a scientific ground for the use of diverse chemopreventive strategies in controlling damage at genetic and molecular level to prevent the ongoing transition of the various oral premalignancy into frank malignancy.

\section{References}

1. Qiang F et al. Nanoparticle Albumin - Bound (NAB) technology is a promising method for anti-cancer drug delivary. Recent patent on anti-cancer drug discovery 2009;4:262-272.

2. Digant G, Pretreatment serum Albumin as a predictor of cacner survival: A systemic review of the epidemiological literature. Nutrition journal 2010;9:69.

3. Abhishek $\mathrm{SN}$ et al. Changing biochemical markers and ongoing process of transformation: - A pilot study. International Journal of Physics and Mathematical Sciences2012;2(1):164-73.

4. Harinder G. Antioxidants in oral cancer prevention. Am J Clin Nutr 1995;62(suppl):1410S-6S.

5. Chapple I, Reactive oxygen species and antioxidants in inflammatroy diseases. J Clin Periodontol 1997;24:287-96.

6. Lannitti T, Palmieri B. Antioxidant therapy and its effectiveness in oxidative stress mediated disorders. In Oxidative stress in Vertebratesand invertebrates. Molecular aspects on cell signaling 2011. 
7. Daniela $\mathrm{M}$ et al. Oxidative stress - A possible link between systemic and oral diseases. Farmacia 2011;59(3):329-37.

8. Carmia B. Dietary antioxidants and human cancer. Integrative Cancer Therapies 2004;3(4):333-341.

9. Johnson NT user and oral cancer: A global perspective. J Dent Edu 2001;65:328-39.

10. Mallikarjuna RK. Salivary Albumin as a Biomarker for Oral Squamous Cell Carcinoma and Chronic Periodonitis. Ann Med Health Sci Res 2017;7:337-340.

11. Priya P, Shanta B, Nagini S. Oxidant antioxidant status in patients with oral squamous cell carcinoma in tissue and plasma - A biochemical analysis on 30 samples. Asian J. Exp. Biol. Sci. 2011;2(2):316-327.

12. Bargavi K. A Ashalatha. G, Venkat B .P, Rajanikanth .AV, Malathi .N S as a diagnostic bio fluid - Review. J Orofac Sci 2010;2(3):66-70.

13. Battino M, Ferreiro MS, Gallardo I, Newman HN, Bullion P. The antixoidant capacity of saliva. J Clin Periodontol 2002;29:189-94.

14. Kaufman E, Lamster IB. The diagnostic application of saliva - A review. Crit Rev Oral Biol Med 2002;13:197-212.

15. Ahishek SN, Mubeen K, K. R. Vijayalakshmi, M. Anitha Serum Albumin: Implications in oral squamous cell carcinoma. Academic Journal of Cancer Research 2011;4(2):56-60.

16. Khanna R. et al. Lipid peroxidation and antioxidant enzyme status in oral carcinoma patients. Kathmandu Univ. Medical Journal 2005;3(12):334-39.

17. Pendyala G, Thomas B, Kumari S. The challenge of antioxidants to free radicals in periodontitis. J Indian Society Periodontol 2008;12:79-83.

18. Valko M, Leibfritz D, Moncol J, Cronin M.T.D., Mazur M., Teser J., Free radicals and antioxidants in normal physiological function and human disease. Int $\mathrm{J}$ Biochen Cell Biol 2006;39:44-84.

19. Mohora M., Greabu M., Totan A., Mitrea N., Battino M., Redox - sensitive signaling factors and antioxidants Farmacia 2009

20. Kolanjiappan K., C.R. Ramachandra and S. Manoharam. Biochemical changes in tumor tissues of oral cancer patients. Clinical Biochemistry 2003;36:61-65.

21. Halliwell B., Reactive species and antioxidants. Redox biology is a fundamental theme of aerobic life. Plant physiol 2006;141:314-22.

22. T. lannitti, V. Rotttigni, B. Palmieri. Role of free radicals and antioxidant defences in oral cavity - related pathologies. J Oral Pathol Med 2012.
23. Cerutti PA, Trump BF. Inflammation and oxidative stress in carcinogenesis. Cancer cells 1991;3:1-7.

24. Niki E. Do free radicals play classical role in the artherosclerosis ? Low density lipoprotein oxidation and vitamin E revisited. J Clin Biochem Nutr 2011;48:3-7.

25. Beevi SSS, Rasheed AMH, Geetha A. Evaluation of oxidative stress and nitric oxide levels in patients with oral cavity cancer. Jpn J Clin Oncol 2004;34:379-385.

26. Storz P. Reactive oxygen species in tumor progression. Front Biosci 2005;10:1881-96.

27. Abhishek SN, Mubeen K, Vijayalakshmi KR, Suman B, Gayathri HC and Anitha M. Serum protein, albumin and advanced oxidation protein products (AOPP) - implication in oral squamous cell carcinoma. Malaysian J Pathol 2012;34(1):47-52

28. Borek C. Antioxidants and cancer. Sci Med (Phila). 1997;4:51-62.

29. J. P. Nicholson, M. R. Wolmarans, G. R. Park. The role of Albumin in critical illness. British Journal of Anaesthesia 2000;85(4):599-610.

30. Moran E.C., A.S. Kamiguti, J.C. Cawley and A.R. Pettitt. Cytoprotective antioxidant activity of serum albumin and autocrine catalase in chronic lymphocytic leukemia. British J. Hematol. 2002;116:316-28.

31. Richter E, Connely R.R, Moul J.W. The role of pre-treatment serum Albumin to predict pathologic stage and recurrence among radical prostatectomy cases. Prostate cancer and Prostate disease 2000;7:186-190.

32. S Gokul, VS Patil, R Jailkhani, K Hallikeri, KK Kattappagari. Oxidant-antioxidant status in blood and tumor tissue of oral squamous cell carcinoma patients. Oral Diseases 2010;16:29-33.

33. Ko W-F, Helzsouer K.J, Comstock G.W. Serum Albumin billirubin and uric acid and anatomic site specific incidence of colon cancer. J Natl Cancer Inst 1986;874-875.

34. Digant G, Christopher G Lis. Pretreatment serum albumin as a predictor of cancer survival: A systematic review of the epidemiological literature. Nutrition Journal 2010;9:69.

35. Battino M, Ferreiro MS, Gallardo I, Newman HN, Bul $\neg$ lion P. The antioxidant capacity of saliva. J Clin Periodontol 2002;29:189-194.

36. Moore S, Calder KAC, Miller NJ, Rice-Evans CA. Antioxidant capacity of saliva and periodontal disease. Free Rad Res 1994;21:417-425.

37. Roche M, Rondeau P, Singh NR, Tarnus E, Bourdon E. The antioxidant properties of serum albumin. FEBS Letts 2008;582:1783-1787.

*Corresponding author:

Dr. Riti Tushar Kanti Sinha, Department of Pathology, ESI-PGIMSR Medical College \& Hospital, Joka, Kolkata, West Bengal, India Phone: +91 9427350988

Email: researchguide86@gmail.com

Financial or other Competing Interests: None. 